\title{
A Compact Test System for Simulating Multipath Interference
}

\author{
Mohammed Eslami' ${ }^{1}$, Howard I. Bassen ${ }^{2}$ \\ ${ }^{1}$ Analytics, Modeling, and Simulation Division, Aptima Inc., Washington DC, USA \\ ${ }^{2}$ Division of Physics, Food and Drug Administration, Silver Spring, USA \\ Email: meslami@aptima.com, Howard.bassen@fda.hhs.gov
}

Received 8 April 2014; revised 17 May 2014; accepted 2 June 2014

Copyright (C) 2014 by authors and Scientific Research Publishing Inc.

This work is licensed under the Creative Commons Attribution International License (CC BY). http://creativecommons.org/licenses/by/4.0/

(c) (i) Open Access

\begin{abstract}
Fading and inter-symbol interference (ISI) arising from multipath effects are a common source of both latency and packet errors in wireless communications. Test methods often require large environments to produce long delays that are enough to affect the communications between a transmitter and receiver. This paper presents a simple, compact test method to produce and isolate effects from multipath interference simulating these effects produced by discrete distances. Signals with controlled delays can be created and combined using multiple antennas in two isolated small ( 2 foot $/ 60 \mathrm{~cm}$ ) cubes constructed with microwave absorber. We demonstrated this with a pulsed RF signal and a signal from an $802.11 \mathrm{n}$ access point with an internal antenna. This method can be further extended to provide a compact test-bed for almost any wireless interference or coexistence test.
\end{abstract}

\section{Keywords}

Fading, Multipath, Wireless Coexistence, Delay Line, Interference

\section{Introduction}

Multipath interference arises from a signal arriving at the receiver from multiple trajectories or paths from the transmitter. These signals with different path lengths from environmental reflectors have two effects: 1 ) in the amplitude domain they cause fading by constructively or destructively interfering with the original (direct path) signal, and 2) in the time domain they cause a delay spread in the accumulated signal. The two effects are due to the differences between the direct and reflected signals in the delay time of arrival of the reflected signals. This results in phase changes which can constructively or destructively interfere with the signal which is maximized if the path length difference is a multiple of a quarter wavelength. Experimental tests in an anechoic chamber using multiple 
antennas [1], and Monte Carlo simulations [2] and have been used to measure the effect of multipath interference in direct path and non-line-of-sight (NLOS) indoor environments.

Orthogonal frequency division multiplexing (OFDM) is a technique used to combat multipath interference [3]. In OFDM the channel frequency is divided into subcarriers of smaller bandwidth than the channel. This reduces the bandwidth of transmitted samples to only a fraction of the whole channel. The higher data rates make it difficult to solve the problem with simple channel equalization, but OFDM solves the problem by sending the data in parallel on orthogonal frequencies. As long as the delay spread of the signal is less than the symbol period on the subcarrier, then the multipath problem is solved fairly well. However, as we are moving to higher data rates, the subcarriers can only allocate so much bandwidth and thus the spread of the signal will again induce ISI. Coexistence studies of other in-band signals with OFDM-based communications were conducted [4]-[6].

Experiments modeling large environments with dimensions of 50 feet or more containing reflecting surfaces are difficult to conduct indoors. This is especially true for environments free from outside radiofrequency (RF) signals. A very costly anechoic chamber of this size would be needed to meet this requirement. In this paper we present a simple method, using a compact test environment, to simulate and measure the multipath effects that arise from reflecting objects in a large environment. In our system, we performed measurements that produced multipath effects in received signals. We used controlled delays of a custom pulse modulated RF source and for $802.11 \mathrm{n}$ signals. This methodology can be further extended to provide a compact test bed for any wireless coexistence test.

This paper is organized as follows: Section II provides an overview of prior work on tests conducted to measure fading and delay spreads arising from multipath interference; Section III introduces the test methodology used in this paper and analyzes its results; and Section IV concludes with a summary and future work.

\section{Prior Work}

\section{Experimental Setups}

Studying the effects of multipath interference is important for determining the degradation of the wireless signal in various environments. Papers that discuss experimental modeling of multipath effects on digital wireless communications often have experiments that are conducted in a location similar in size to the place the devices are to be deployed. In addition, there are numerous computational studies of experiments conducted to analyze the effect of multipath interference.

In [7] a non-line-of-sight (NLOS) setup was tested with two Zigbee (802.15.4) nodes and it was determined that in typical indoor environments that effects from the delay spread of the signal can be neglected at those low data rates. As a matter of fact, protocols that are using anything lower than $50 \mathrm{Mb} / \mathrm{sec}$ can have the effects of delay spread ignored in typical indoor environments (transmitter to receiver distance less than $50 \mathrm{~m}$ ). However signal loss due to fading effects from multipath still exists at short distances. The NLOS study was performed to find the largest distance where a $0 \%$ packet error rate (PER) was maintained between the transmitter and the receiver. Once the location was identified, the interferer, an 802.11 source, was brought in the vicinity of the victim, an 802.15.4 node, to analyze its effects.

In [8] delay spreads on the order of 10 - 40 ns are reported in a typical office environment $\left(6^{\text {th }}\right.$ floor of Faraday Tower in University of Wales) filled with filing cabinets and other furniture by sweeping the carrier frequency from 2 - $20 \mathrm{GHz}$. They measured frequency responses with three different transmitter locations and 180 distinct receiver locations to model various paths present between the transmitter and receiver which corresponded to a multipath signal whose amplitude was $1 / 3$ the LOS signal delayed by $10 \mathrm{~ns}$. In an experiment by [9] to estimate the effects of multipath on "chaotic radio" pulse tests were conducted in corridors with dimensions of $4 \mathrm{~m} \times 40 \mathrm{~m} \times 3$ $\mathrm{m}$ and conference halls of $20 \mathrm{~m} \times 4 \mathrm{~m} \times 16 \mathrm{~m}$ by varying the distance between the transmitter and receiver from 1 $-20 \mathrm{~m}$. After conducting these tests in these various environments, their results showed that the effect of multipath interference on their signal was negligible. In [10] multipath effects are measured in various large environments such as a multi-story apartment building, an oil refinery, a long corridor in an office building typical of many commercial facilities, and a subterranean tunnel, to characterize the risk of BER to emergency responders.

These experiments and other similar experiments on measuring effects of multipath were conducted in large environments whose sizes ( $>20 \mathrm{~m}$ ) were needed to analyze delay spreads. For $802.11 \mathrm{n}$ and other technologies the separation distance between 2 nodes can be much greater. The experimental setup provided in this paper demonstrates the ability to model the effect of large delay spreads and multipath fading in a compact, isolated manner. These normally require large shielded spaces with reflecting clutter, but we developed a method to do 
this in an area that is less than several square meters.

Other smaller environments have been investigated for this purpose but each has their drawbacks. For instance, [11] [12] uses a reverberation chamber to simulate different multipath propagation environments with different loads, antenna positions, etc. A comprehensive experimental study by [13] studied the wireless coexistence and EMC of Bluetooth and 802.11b in a Gigahertz Transverse Electromagnetic (GTEM) cell. Data Integrity and throughput were measured during various forms of interferences. GTEM cells are bulky, expensive devices and focus only on TEM waves, while in real environments many modes of TEM, TE, and TM waves could be present. It is for these reasons that a method was developed in this paper to mimic a real environment as closely as possible while providing a tester with explicit control of various isolated paths that they want to examine.

\section{Multipath Test Methodology and Results}

\subsection{Test Methodology}

Large test environments are needed to generate long delay spreads. For instance, a path difference of $20 \mathrm{~m}$ between two waves leads to a 66.7 ns delay in their arrival time at the receiver. When modeling large indoor environments, it is difficult to produce and isolate the effects of multipath interference unless RF absorber is used to line the test environment and cover any metallic cables or other miscellaneous conducting objects. Our goal in generating experimental modeling of multipath interference is to selectively produce, in a very small and low cost environment, a delay between the times of arrival of two or more signals at the receiving device under test (DUT). This test methodology works explicitly well for DUTs that have integrated antennas that lack a cable connector, like many medical devices. This is to see the effects of fading during constructive and destructive interferences of the reflected signals. The novelty in this method is that we can mimic delays that are produced from large environments in a small, low-cost setup. The various path differences can be selectively analyzed to find the path that would produce the worst case interference and result in EMI of the transmitter with the DUT.

The model of large environments was conducted by isolating the transmitting DUT and receiving DUT from one another by placing them in separate $61 \mathrm{~cm} \times 61 \mathrm{~cm}$ shielded enclosures lined with absorbing material. This avoids any coupling of external fields. It also allows control of the RF signals at the receiver by tapping into the radiating transmitter's signals with an antenna. The use of one or more long coaxial cables as delay lines can provide the appropriate arrival times of multiple signal paths that would arise from multipath effects. Attenuators and amplifiers are placed within the setup to ensure appropriate signal strength at the various stages of the test-bed.

The test setup used in our lab is shown in Figure 1 and only focuses on a direct path and one delayed path, however, it should be noted that this setup can be expanded to model any environment for wireless coexistence tests.

The system's signal flow is as follows.

1. RF transmissions from the transmitting DUT are captured by antenna R1 in absorber lined enclosure with metal backing (E1).

2. Antenna R1 delivers a signal to an RF power splitter via coaxial cable. The splitter divides the signal and delivers it to an attenuator and to a delay line (low loss coaxial cable).

3. The attenuator and delay line signal outputs are routed via coaxial cables to absorber lined enclosure with metal backing (E2) containing transmitting antennas.

4. The attenuator reduces the direct-signal to transmitting-antenna T1 to a selected amplitude. The other output of the splitter is routed to the delay line whose output is routed to antenna T2.

5. In enclosure (E2) antenna T1 transmits a direct path signal to receiving antenna R2 which is separated by distance L1. The delay line establishes a later time of arrival for a second signal radiated from antenna T2 and is separated from antenna T1 by an absorbing divider. T2 is separated from antenna R2 by distance L1 plus distance L2.

6. The distance L2 can be varied to produce constructive or destructive interference with the direct and delayed signals arriving at R2.

7. Receiving antenna R2 delivers a signal to a coaxial cable from enclosure E2 to enclosure E3. E3 shields other outside sources from antenna T3. This antenna radiates a wireless signal to the receiving DUT and isolates the antenna setup in E2 from any reflections arising from the DUT.

In step 4 of the signal flow listed above; the attenuator is adjusted to model various far field transmission path 


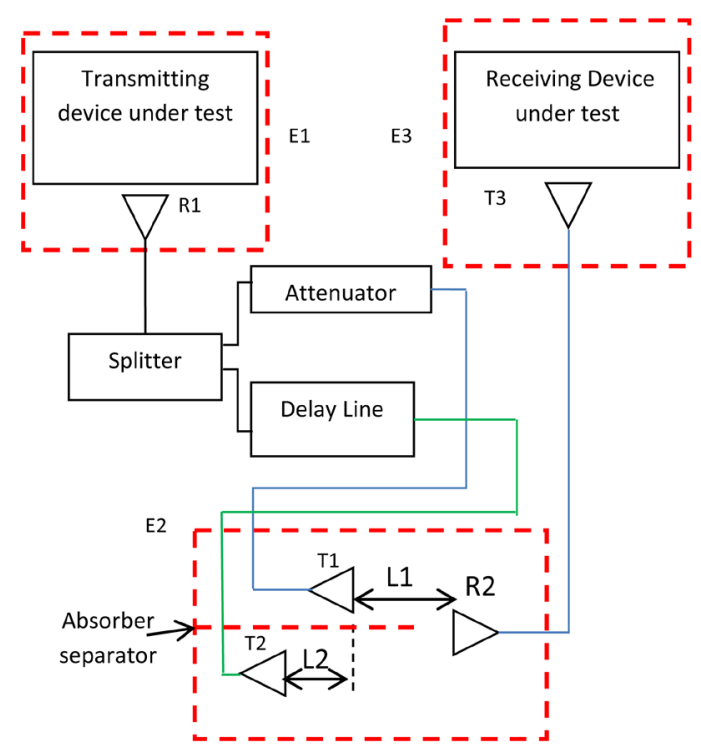

Figure 1. Red dotted lines indicate absorbing enclosures (E1, E2, and E3) that contain receiving antennas (R2) and transmitting antennas (T1, T2, T3). Antennas $\mathrm{T} 1$ and $\mathrm{T} 2$ are also separated by an absorber to avoid mutual coupling and reflections.

loss conditions. One condition simulates the direct path loss between the wireless DUT when the receiving DUT is in the far field of the transmitting device. This loss $\left(\mathrm{P}_{\mathrm{L}}\right)$ between the transmitting and receiving DUT is given in Equation (1).

$$
\mathrm{P}_{\mathrm{L}}=\left[\mathrm{G}_{\mathrm{TX}} * \mathrm{G}_{\mathrm{RX}} * \lambda^{2}\right] / 4 \pi \mathrm{R}
$$

$\mathrm{R}$ is the distance between the transmitting and receiving antennas. The carrier signal wavelength is $\lambda$ and $\mathrm{G}_{\mathrm{TX}}$ and $\mathrm{G}_{\mathrm{RX}}$ are the gains of the transmitting and receiving antennas respectively.

The delay line can be chosen to adjust the delay between the direct signal (antennas T1 and R2) and the signal traveling a different path (reflected multipath). Although not shown in Figure 1, the signal can be further split into more delay lines and antennas to model more trajectories. Using a single delay line can determine the trajectory that will have the greatest effect on the direct signal. The delay line does not necessarily incorporate the worst case scenario of multipath fading. In order for the worst case scenario to be analyzed, an additional variable delay whose size is no larger than half wavelength must be inserted to find the maximum and minimum voltages of the incident wave. By moving antenna T2 various distances (L2) greater or equal to plus or minus one quarter wavelength, one can measure the effects of constructive and destructive interference when the signal arrives in and out of phase, respectively. Angle $\varphi$ is the phase shift between the direct and delayed wave. This procedure is qualitatively shown in Figure 2.

\subsection{Measurements Using an RF Signal with Pulsed Modulation}

In an effort to see the effects of fading clearly, the following multipath setup was used instead of an actual wireless signal. A pulse-amplitude modulated continuous wave signal at $2.45 \mathrm{GHz}$ was produced using an Agilent MXG N5183A Signal Generator that provided a peak power signal at $2.45 \mathrm{GHz}$ of $4.00 \mathrm{dBm}$. The pulse modulation was provided by an HP 8007A pulse generator. The signal generator's output was split by a Mini-Circuits ZN2PD2-50-S+ splitter that provides a signal for the direct path and the delayed path. The direct signal was first passed through a $-10 \mathrm{~dB}$ attenuator (Narda 757C) and then fed to antenna $\mathrm{T} 1$ to equalize the signal strength with respect to the signal out of the delayed signal. A single low loss coaxial cable, $50 \mathrm{~m}$ in length (Pasternack Enterprises PE37940-1968.25) formed the delay line whose total measured loss was $-35 \mathrm{~dB}$.

The setup is shown in Figure 3. Antennas T1, T2, and R2 are all +6 dB gain Pasternack Enterprises PE51086 sleeve dipole antennas. Antenna R2 received the signals from T1 and T2 and passed on the sum to an HP 8449B 


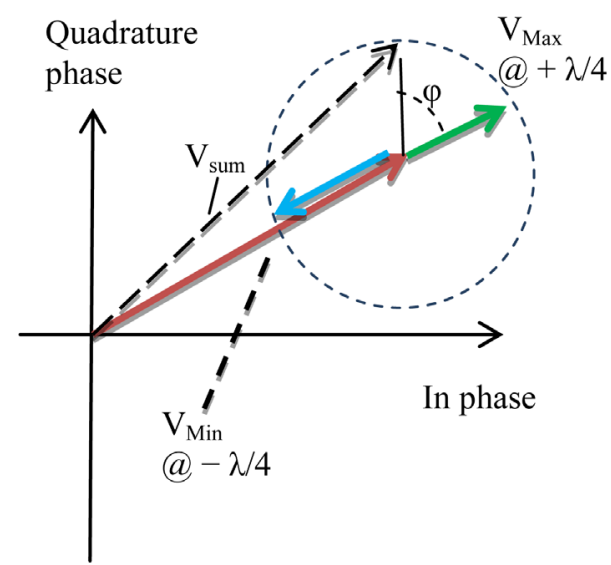

Figure 2. Initial (red) LOS phasor with the single path reflection received. The reflection is shown rotated to (blue) minimum and (green) maximum effect. The vector sum $\left(\mathrm{V}_{\text {sum }}\right)$ of the red vector with the blue/green vector gives the overall magnitude of the phasor.

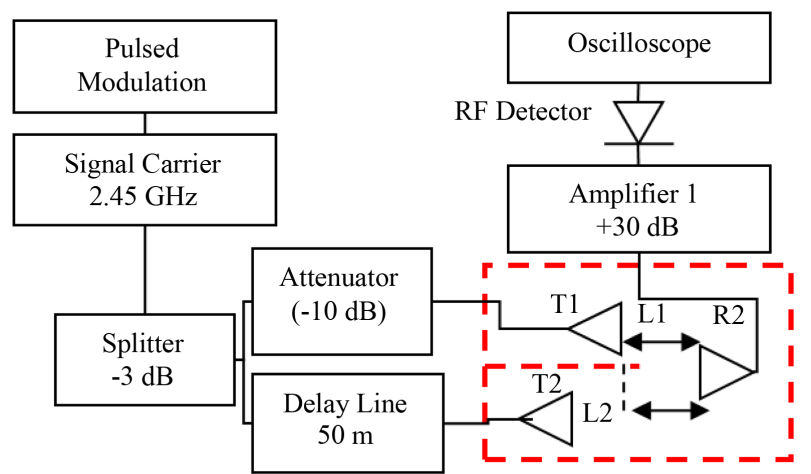

Figure 3. Test setup to measure fading effects from $50 \mathrm{~m}$ delay line with pulsed modulation on a $2.45 \mathrm{GHz}$ carrier signal. The attenuator is modeling the direct path while the delay line introduces a $50 \mathrm{~m}$ path difference. All antennas are sleeve dipole antennas at $+6 \mathrm{~dB}$ gain and the red dashed box is an absorbing enclosure.

Preamplifier (+30 dB). The amplifier fed the Advanced Control Components, ACSP-2538NC3R RF envelope detectors with enough power so their output could be resolved by an Agilent Technologies Infiniium DSO91048 Digital Storage Oscilloscope.

The separation distance (L1) between antenna T1 and antenna R2 was arbitrarily fixed at $41 \mathrm{~cm}$ to provide a baseline measurement. The delayed signal passed through a $50 \mathrm{~m}$ spindle of cable which was then fed to antenna T2, whose distance (L2) was varied from $41 \mathrm{~cm} \pm \lambda / 4(3.1 \mathrm{~cm})$. Before testing the setup in Figure 3, preliminary measurements were first made to ensure the delayed pulse could be captured by the RF detectors. The setup used to make these measurements was identical to the setup shown in Figure 3 except that the lines going into antennas $\mathrm{T} 1$ and $\mathrm{T} 2$ were directly connected to two RF detectors (one positive polarity and one negative polarity) and then to the oscilloscope. Figure 4 shows the outputs of the RF envelope detectors with a signal directly from the RF signal generator and splitter (no antennas). Outputs are the direct (yellow-top) and delay line (green-bottom) having a delay on the order of $217 \mathrm{~ns}$. The signal generator pulse width is $1.22 \mu \mathrm{sec}$.

For the setup shown in Figure 3, it was expected that the pulse would show some sort of abrupt change after about $205 \mathrm{~ns}$, if the delay was assumed to only be a result of the $50 \mathrm{~m}$ delay line. The extra $12 \mathrm{~ns}$ arises from measurement errors in reading the oscilloscope, and delays introduced by the connecting cables, amplifiers, and attenuators. Antenna T2 (connected to the delay line) was moved backwards and forwards roughly a distance of 


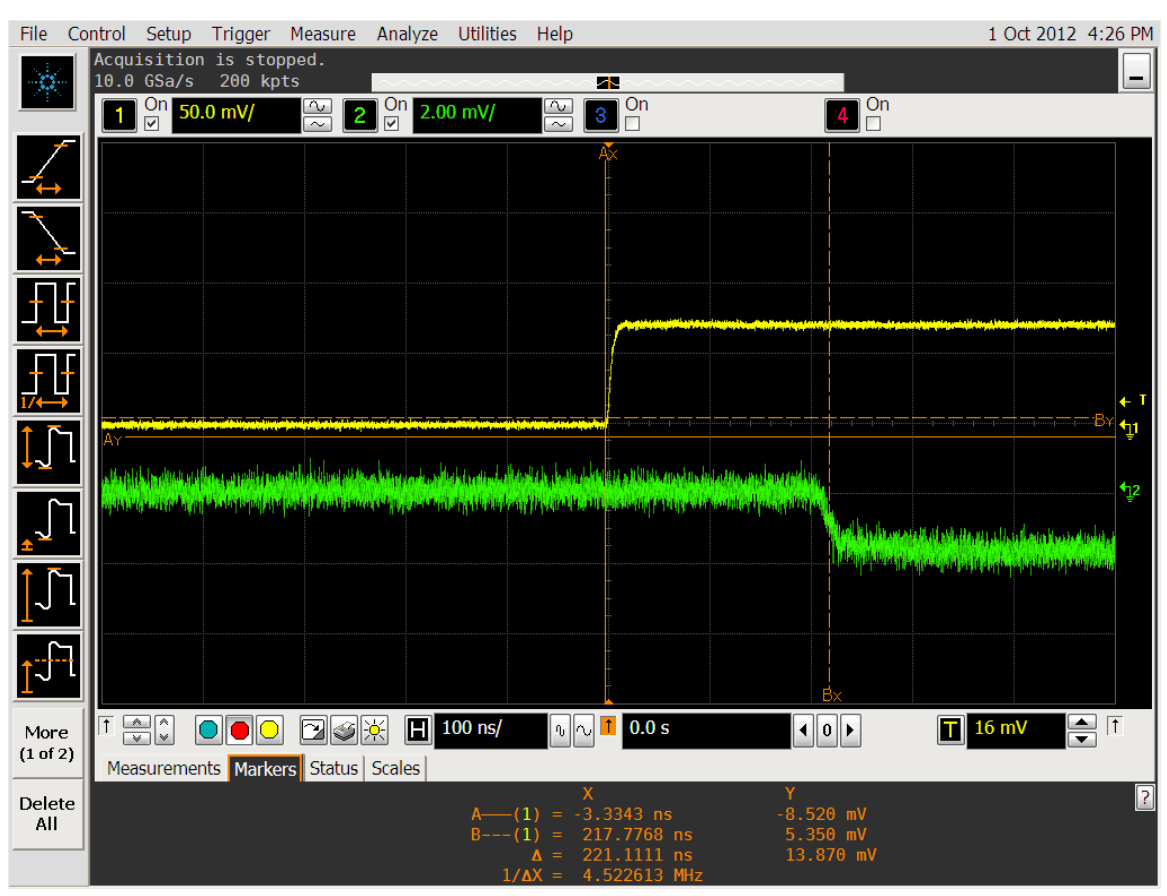

Figure 4. Output from the (yellow-top) direct/positive and (green-bottom) delayed/negative detectors with a pulse width set to $1.2 \mu \mathrm{sec}$. The delay observed is on the order of $217 \mathrm{nsec}$.

about $[-\lambda / 4, \lambda / 4]$ with respect to antenna T1. This changed the phase with respect to antenna T1 and constructive or destructive interference with the direct signal occurred as seen by receiving antenna R2. Figure 5 shows the results with the interference occurring at about $210 \mathrm{~ns}$ after the pulse begins. The antenna was moved back and forth until a maximum ratio $(\rho)$ was found for each case.

$$
\rho=\frac{\text { Interefered Height }}{\text { Normal Height }}
$$

Finally, we can explore the use of our small, inexpensive enclosures to eliminate the need for an expensive, large anechoic chamber. These enclosures can be applied to several types of wireless coexistence and EMC tests. The enclosures provide the ability to test wireless receivers without interference from other ambient RF sources. They can be used with a pickup antenna inside them to couple and adjust the amplitude and pulse timing of radiated RF emitted by a simultaneous mix of medical and other wireless devices with embedded antennas and no coaxial output connector.

In the case of constructive interference it was determined that $\rho=0.92$, and in the case of destructive interference we saw that $\rho=0.42$. The change in field strength levels at the two distances attributes to the difference in these ratios. We noted that the amplitudes did not match the system diagram losses shown in Figure 3 . When signals from antennas T1 and T2 coincided, it produced an increase in the waveform ("Interfered Height” in Figure 5(a)). The amount of additional signal was much higher than is predicted by linear systems analysis. However, at the end of the waveform, when only the signal from T2 ("Delay alone") is present, the amplitude of the waveform was as predicted. Numerous checks were performed to identify the cause of this discrepancy. Namely, the end of the waveform seemed to have been produced from a linear system, while the beginning of the waveform was produced by a non-linear one. We performed detailed investigations of possible radiated coupling between the two transmitting antennas, and other sources of signal crosstalk. This did not reveal the source of the increased signal strength from the combined signals. We then evaluated the setup by replacing the antennas with direct connections and a phase shifter. The source of the increased signal was identified. The mixing efficiency of the diode and signal combiner was much higher when the strong Direct Signal and the weaker Delayed Signal appear together. This was observed for different models of detector diodes and with the delay line replaced by a 30 $\mathrm{dB}$ attenuator. 


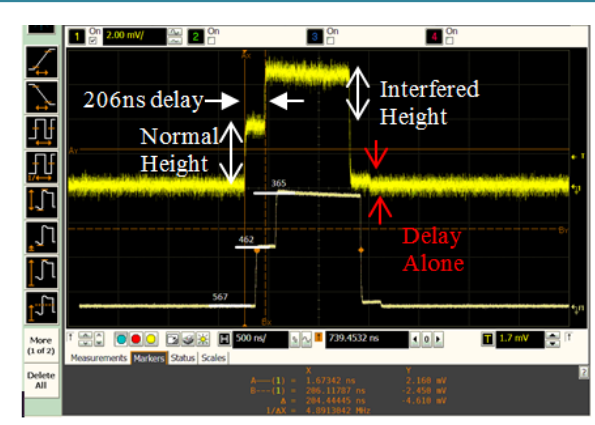

(a)

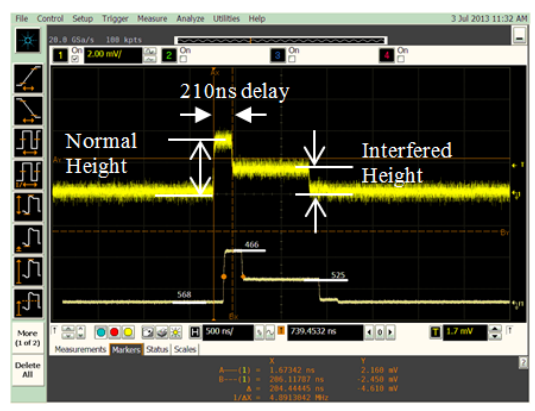

(b)

Figure 5. Measurements taken by moving antenna T2 to (a) $38.2 \mathrm{~cm}$ (roughly $-\lambda / 4$ with respect to antenna T1) for a constructive maximum and (b) $43 \mathrm{~cm}$ (roughly $+\lambda / 4$ with respect to antenna T1) for a destructive minimum. Horizontal arrows indicate that the interference occurs at 206 ns from the start of the pulse as was expected from Figure 4. The white plots depict the average of the raw signal to filter out the high frequency noise and calculate the ratio $\rho$ using the pixel locations of the zero, direct, and delayed signal levels.

\subsection{Measurements Using an 802.11 n Router}

In the previous section, the incoming modulation of the carrier wave was regular and known. Any change in the envelope was easily detected and identified as multipath interference. For the case of an $802.11 \mathrm{n}$ router, the signal was more complex with signals consisting of a series of short pulses within each RF burst. An experiment was conducted to see the effects on the temporal signal at the receiver from an $802.11 \mathrm{n}$ transmitter in the beaconing mode. Packets from a Belkin 450N DB 802.11 n router were first analyzed to form a baseline of common beaconing signals emitted by it while located in an anechoic chamber as shown in Figure 6. The voltages of the measurements that were made are shown in Figure 7.

To study multipath effects on this signal using the delay line, the test setup in Figure 3 was modified and the new setup is shown in Figure 8. Due to the $-35 \mathrm{~dB}$ loss in the delay line, the delayed signal was first passed through a Mini Circuits ZHL-42 RF amplifier to ensure the signal is comparable to that of the direct line to be able to observe effects. In addition, a $60 \mathrm{~Hz}$ noise suppressor was added in series with the delay line to eliminate ground loop noise from the oscilloscope and other AC powered components

This system was used to see if multipath effects were evident. Using discrete movements of antenna T2 from antenna T1 at $0 \mathrm{~cm}, 3 \mathrm{~cm}$, and $17.5 \mathrm{~cm}$, the effects of multipath interference were measured. An example of a comparison of envelope-detected waveforms is shown in Figure 9. One waveform is from the combined signals (direct plus delayed shown in yellow) received by antenna R2 followed by an RF amplifier. The other signal is from the router only (reference shown in green) from antenna R1. The red oscilloscope cursors align the bumps from both signals and show a minor 20 ns delay between the two paths. This is due to the slightly different paths the reference and the direct signal traverse. The delayed signal arrives about $205 \mathrm{~ns}$ after the start of the reference signal; following this, the received combined signal's waveform is notably different (with wider pulses) than the reference signal. The first $200 \mathrm{~ns}$ of the signal are shown with the white vertical lines. The lower amplitude during the first few pulses of the combined signal (yellow) is due to the use of a RF amplifier (Amp 2) making the delayed signal stronger than the direct signal.

\subsection{Comparison of Results}

We compared the two configurations, one consisting of a pulsed continuous wave signal at $2.45 \mathrm{GHz}$ delivered to a 50 meter delay line plus an antenna, as well as a second antenna fed by the signal without delay (Figure 3). Changing the delayed antenna's position yielded a minimum to maximum signal amplitude ratio at the receive antenna. For constructive vs. destructive interference the ratios were 0.92 and 0.42 respectively for the greatest and least interactions. For the $802.11 \mathrm{n}$ router experiment, we could not obtain a simple minimum and maximum amplitude value due to constructive vs. destructive interference. This is because the waveform consists of nonuniform bursts with a duration of more than 100 nsec (Figure 9). Our delay line produced 200 nsec delays so changes in amplitude due to interference are not possible to find. Therefore to evaluate the effects of multipath on this signal, the reduction of the quality of data transmission should be measured (packet error rate). This is 


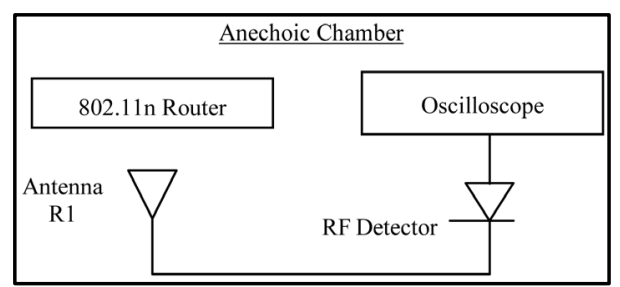

Figure 6. Test setup to determine the baseline signals from an $802.11 \mathrm{n}$ router. The actual signals from this detector can be seen in Figure 7.

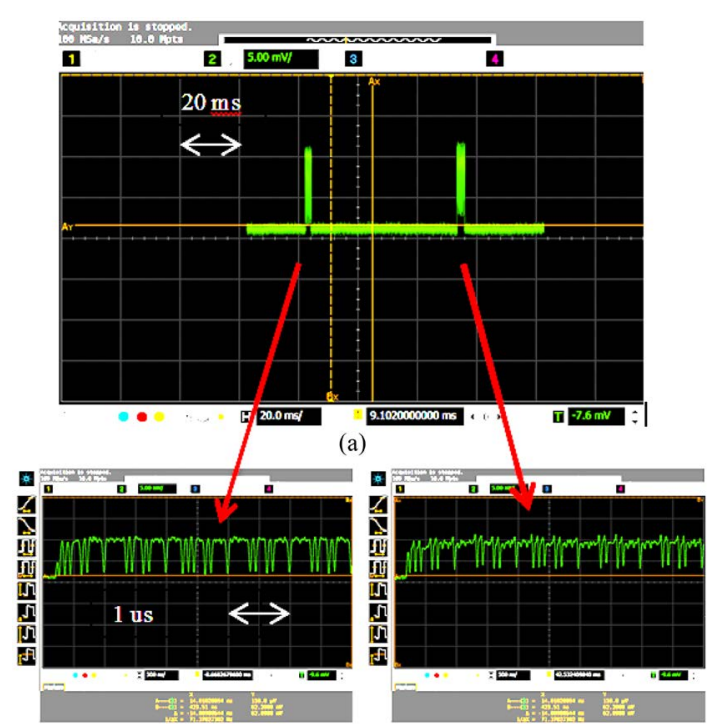

(b)

(c)

Figure 7. (a) Beaconing packets from an $802.11 \mathrm{n}$ router (b) Zoomed in on first pulse and (c) Zoomed in on second pulse.

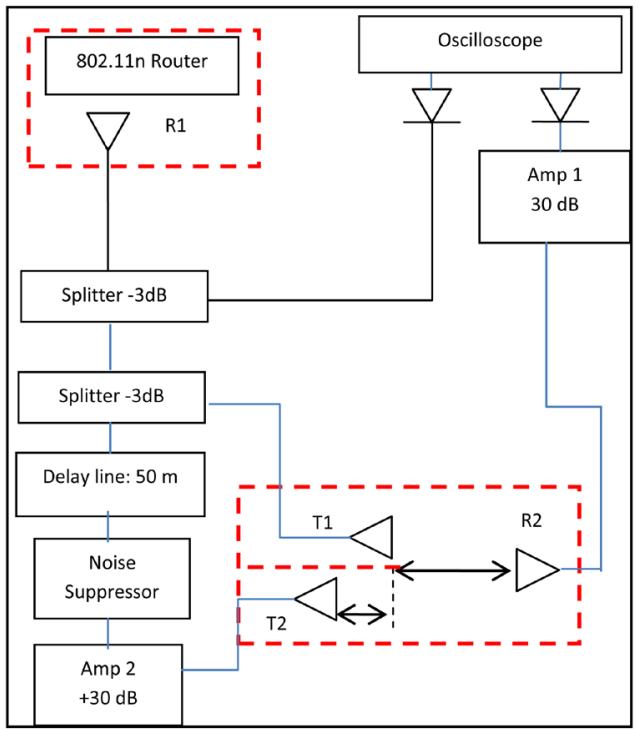

Figure 8. Experimental setup for multipath interference modeling with $802.11 \mathrm{n}$ router signal being tapped by a sleeve dipole antenna R1. All antennas and splitters are identical to the previous setup. 


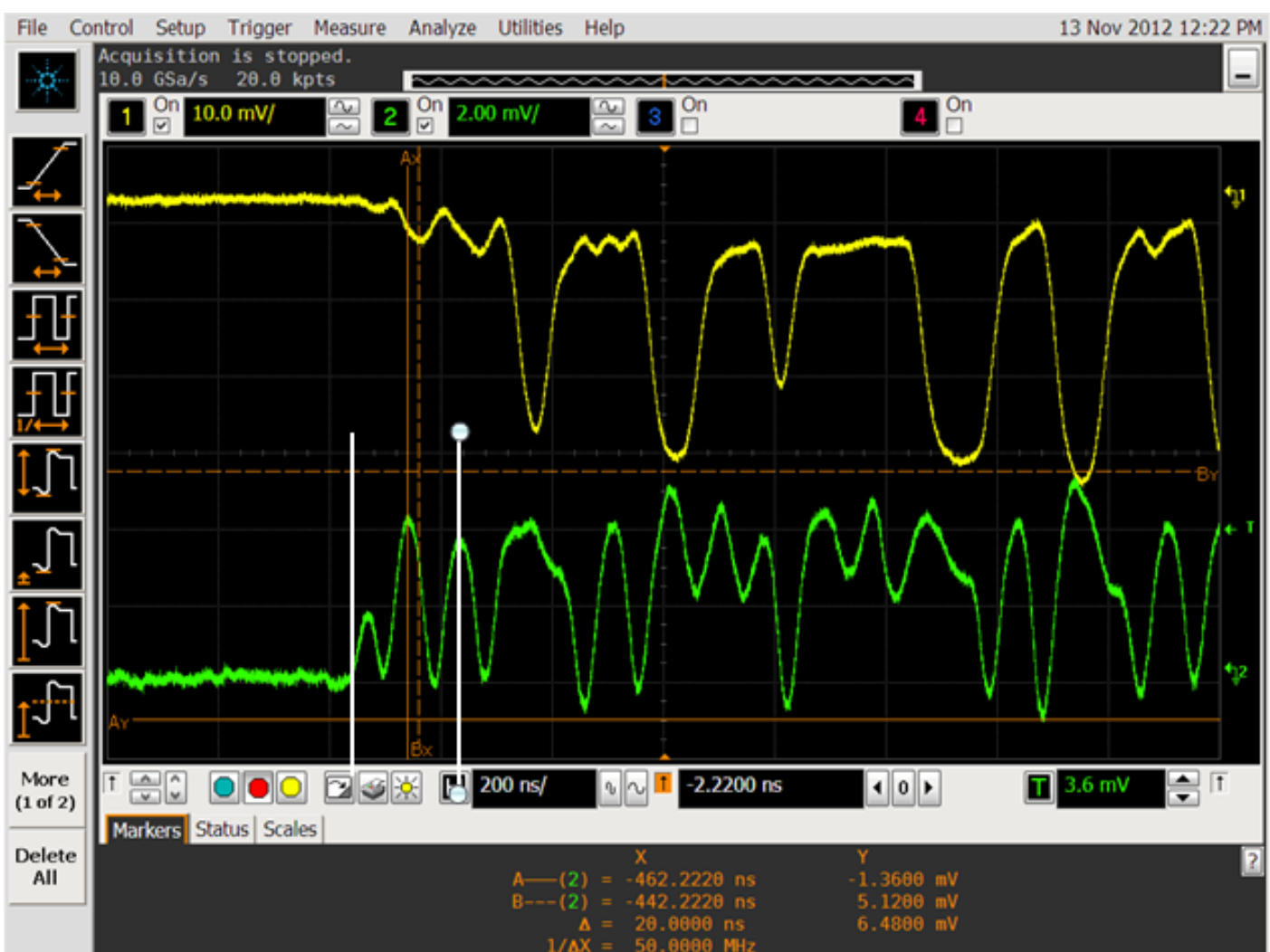

(a)

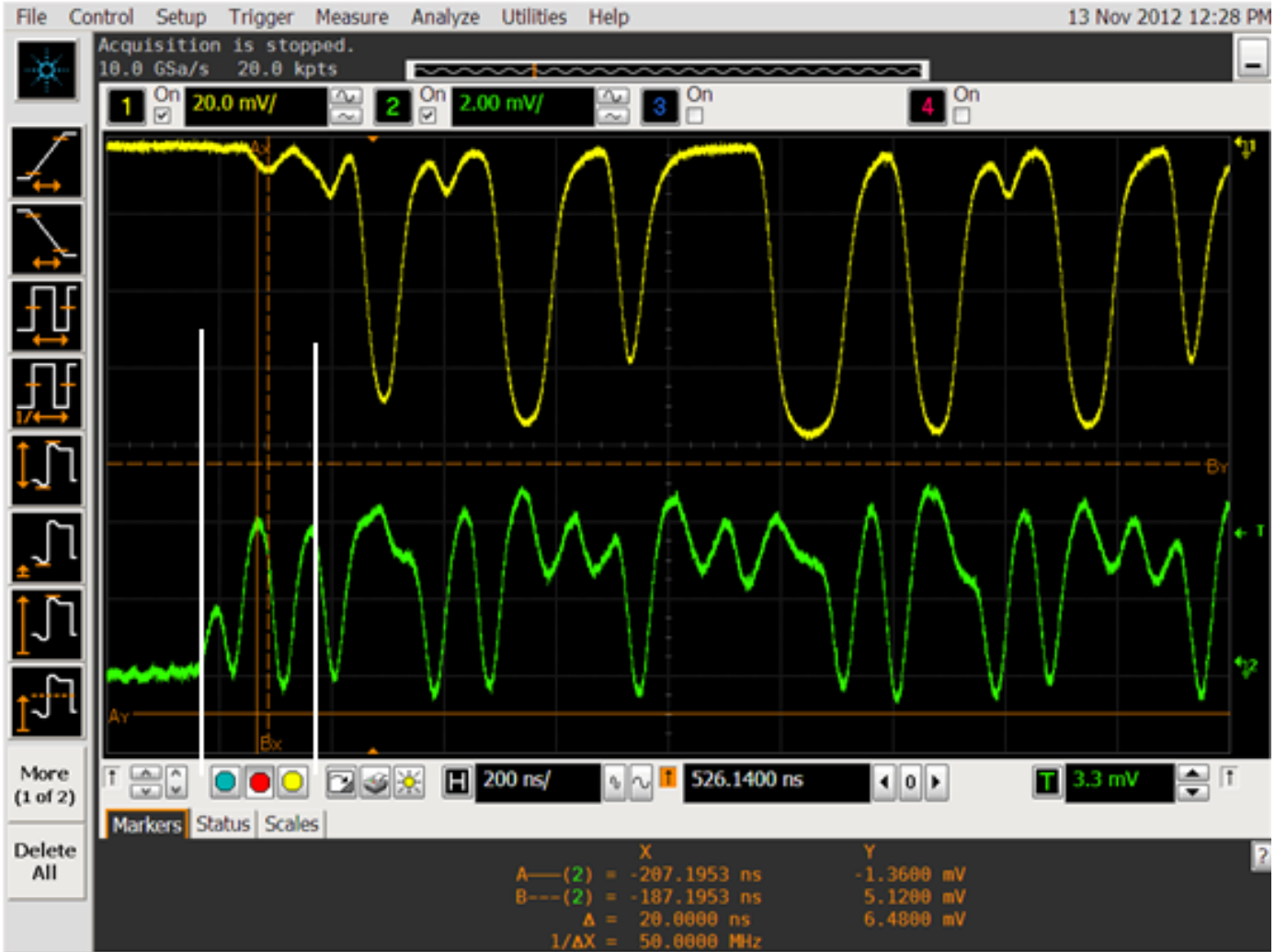

(b) 


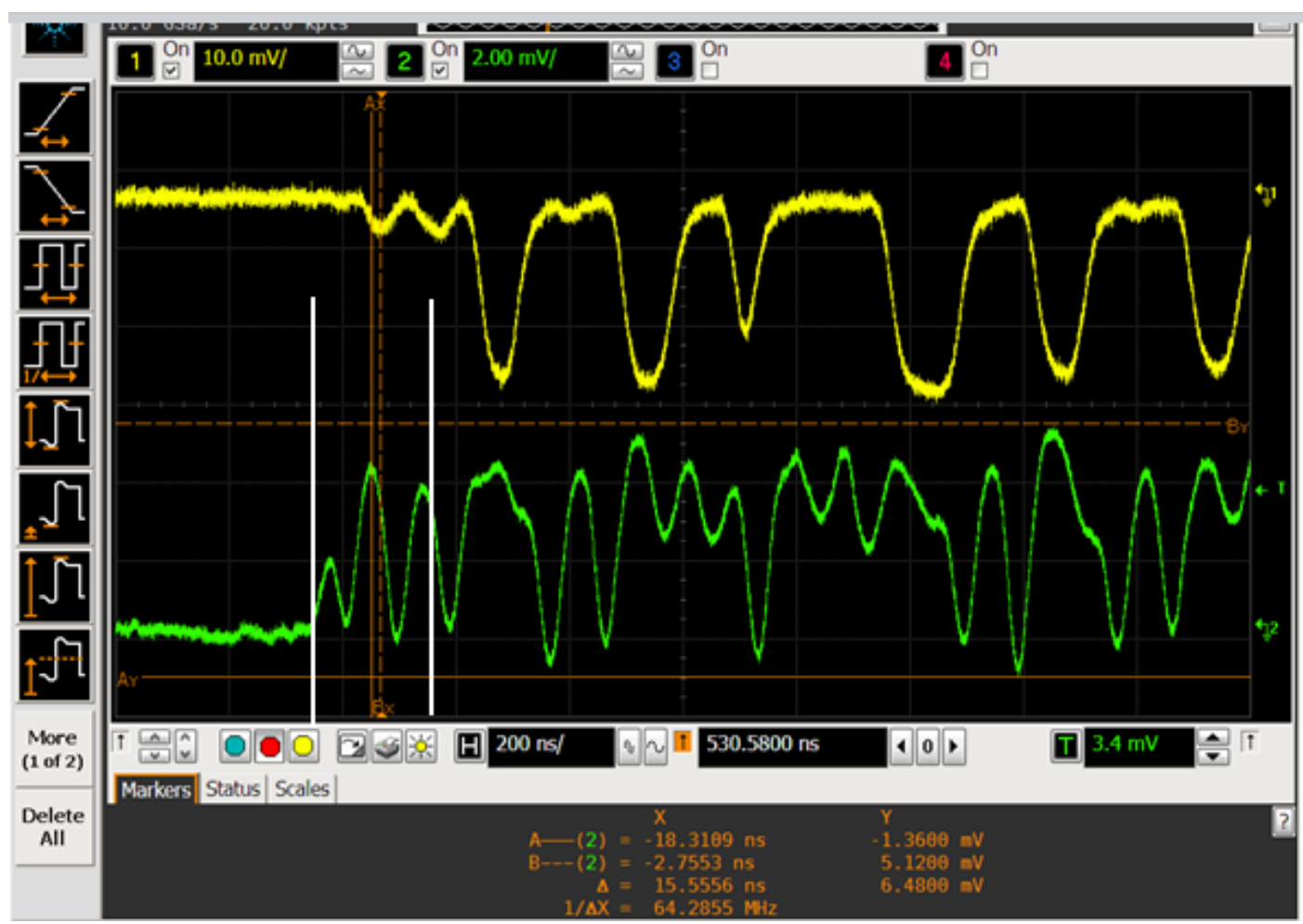

(c)

Figure 9. Measurements from an $802.11 \mathrm{n}$ router signal in the configuration shown in Figure 8 with the delayed antenna placed at (a) $0 \mathrm{~cm}$ (b) $3 \mathrm{~cm}$ (c) $17.5 \mathrm{~cm}$. The yellow signal depicts the sum of the direct and delayed antenna as it is received in R2, while the green shows a direct line from antenna R1. The red vertical cursors align corresponding bumps, the slight delay of $20 \mathrm{~ns}$ is due to the different components the signals must traverse. It is clear that after $200 \mathrm{~ns}$, shown by the white vertical overlays, the signals no longer have corresponding forms since the delayed antenna, T2, interferes with the direct signal from T1.

the next step in our future research.

\section{Conclusion}

In this paper, we explored a method for experimentally simulating multipath effects normally occurring in large, reflecting environments. The simple, compact test methodology can also be conducted in places where large anechoic chambers are not readily available. We used various configurations: each consisting of several compact, absorber-lined enclosures, a coaxial cable delay line (50 m long), a number of low cost transmitting and receiving antennas, a RF amplifier, and other low cost components. Effects on the output signal were observed by moving the delayed transmitting signal antenna in discrete steps between $[-\lambda / 4, \lambda / 4]$ while the transmitting antenna with the non-delayed signal remained stationary. The overall experimental setup provided a single 205 ns multipath-like delay in a $1.5 \mathrm{~m} \times 3 \mathrm{~m}$ area instead of a $50 \mathrm{~m}$ long room or anechoic chamber.

\section{Future Work}

The next steps for this experimental setup are to analyze the effects of the simulated multipath interference in the digital network domain. Evaluating network packets down to the symbol or bit level and correlating this with the time domain signal should be performed. Various forms of digital transmissions would be used in these tests. This would provide the ability to compare effects we saw in the time domain with the packet error rate in the network domain. The delay spread in our setup can cause inter symbol interference (ISI), where one symbol overlaps the next in the time domain. Our test system has the potential to create ISI in wireless networks with high bit-rate protocols and non-optimal multipath correction. The $802.11 \mathrm{~g}$ and future protocols can operate at rates of 54 $\mathrm{Mb} / \mathrm{sec}$ or higher and thus may be affected by ISI from multipath interference from a group of large delay spreads. 
Finally, we can explore the use of our small, inexpensive enclosures to eliminate the need for an expensive, large anechoic chamber for other applications. These enclosures can be applied to several types of wireless coexistence and EMC tests. The enclosures provide the ability to test wireless receivers without interference from other ambient RF sources. They can be used with a pickup antenna inside them to couple and adjust the amplitude and pulse timing of radiated RF emitted by a simultaneous mix of medical and other wireless devices with embedded antennas and no coaxial output connector. In the future we can produce a number of simultaneous radiated signals, using multi-way splitters. Each splitter output could feed longer delay lines and individual transmitting antennas, placed in the same enclosure. Each delay line could be composed of a series of several discrete, long spools of coaxial cable, with amplifiers inserted as needed. This test methodology can be used to generate a simple, inexpensive, and compact multipath test-bed for wireless coexistence tests. In addition, once this method is characterized at the bit level for wireless communications, a similar method would be to use programmable delay lines and combiners rather than antennas and splitters. This could prove to be more controllable than that of the current methodology. This approach would be a combination of this test methodology with that proposed in [14].

Finally, we can explore the use of our small, inexpensive enclosures to eliminate the need for an expensive, large anechoic chamber for other applications. These enclosures can be applied to several types of wireless coexistence and EMC tests. The enclosures provide the ability to test wireless receivers without interference from other ambient RF sources. They can be used with a pickup antenna inside them to couple and adjust the amplitude and pulse timing of radiated RF emitted by a simultaneous mix of medical and other wireless devices with embedded antennas and no coaxial output connector.

\section{Acknowledgements}

This project was supported in part by an appointment to the Research Participation Program at the Center for Devices and Radiological Health administered by the Oak Ridge Institute for Science and Education through an interagency agreement between the US Department of Energy and the US Food and Drug Administration.

\section{Disclaimer}

The mention of commercial products, their sources, or their use in connection with material reported herein is not to be construed as either an actual or implied endorsement of such products by the Department of Health and Human Services.

\section{References}

[1] Moschitta, A., Macii, D., Trenti, F., Dalpez, S. and Bozzoli, A. (2012) Characterization of a Geometrical Wireless Signal Propagation Model for Indoor Ranging Techniques. 2012 IEEE International Instrumentation and Measurement Technology Conference (I2MTC), 13-16 May 2012, 2598-2603.

[2] Salih Alj, Y., Despins, C. and Affes, S. (2008) Impact of Multipath Interference on the Performance of an UWB Fast Acquisition System for Ranging in an Indoor Wireless Channel. 6th Annual Communication Networks and Services Research Conference, CNSR 2008, 5-8 May 2008, 390-396.

[3] Rappaport, T. (2002) Wireless Communications. 2nd Edition, Prentice Hall.

[4] Li, Z., Zou, W.-X. and Li, B. (2011) Analysis on Coexistence of Ultra Wideband with OFDM-Based Communication Systems. IEEE Transactions on Electromagnetic Compatibility, 53, 823-830. http://dx.doi.org/10.1109/TEMC.2011.2159979

[5] Sharma, G.V.V. and Srinivasan, S.H. (2005) Symbol Detection in CDMA-OFDM Coexistence. IEEE 16th International Symposium on Personal, Indoor and Mobile Radio Communications, 2531-2534.

[6] Jo, H.-S., Yoon, H.-G., Lim, J., Chung, W.-G., Yook, J.-G. and Park, H.-K. (2006) The Coexistence of OFDM-Based Systems beyond 3G with Fixed Service Microwave System. Journal of Communications and Networks, 8, 187-193. http://dx.doi.org/10.1109/JCN.2006.6182747

[7] LaSorte, N.J., Rajab, S.A. and Refai, H.H. (2012) Experimental Assessment of Wireless Coexistence for 802.15 .4 in the Presence of 802.11g/n. 2012 IEEE International Symposium on Electromagnetic Compatibility (EMC), 6-10 August 2012, 473-479.

[8] Nobles, P. and Halsall, F. (1997) Delay Spread and Received Power Measurements within a Building at 2 GHz, 5 GHz and $17 \mathrm{GHz}$. Antennas and Propagation, Tenth International Conference on (Conference Publication No. 436), 2, 1417 April 1997, 319-324. 
[9] Kuzmin, L.V., Starkov, S.O. and Kletzov, A.V. (2009) Performance of Wireless Communication System with Ultrawideband Chaotic Signals in the Multipath Channel. Progress in Electromagnetics Research Symposium Proceedings, Moscow, 18-21 August 2009, 1493-1497.

[10] Remley, K.A., Koepke, G., Holloway, C.L., Grosvenor, C., Camell, D., Ladbury, J., Johnk, R.T. and Young, W.F. (2009) Radio Wave Propagation into Large Building Structures, Part 2, Characterization of Multipath. IEEE Transactions on Antennas Propagation, 58, 1290-1301.

[11] Genender, E., Holloway, C.L., Remley, K.A., Ladbury, J., Koepke, G. and Garbe, H. (2008) Use of Reverberation Chamber to Simulate the Power Delay Profile of a Wireless Environment. 2008 International Symposium on Electromagnetic Compatibility-EMC Europe, 8-12 September 2008, 1-6.

[12] Fielitz, H., Remley, K.A., Holloway, C.L., Zhang, Q., Wu, Q. and Matolak, D.W. (2010) Reverberation-Chamber Test Environment for Outdoor Urban Wireless Propagation Studies. IEEE Antennas and Wireless Propagation Letters, 9, 52-56.

[13] Seidman, S., Kainz, W., Ruggera, P. and Mendoza, G. (2011) Wireless Coexistence and EMC of Bluetooth and 802.11b Devices in Controlled Laboratory Settings. Open Biomedical Engineering Journal, 5, 74-82.

[14] Howitt, I. and Shukla, A. (2008) Coexistence Empirical Study and Analytical Model for Low-Rate WPAN and IEEE 802.11b. IEEE Wireless Communication and Networking Conference, 31 March-4 April 2008. 
Scientific Research Publishing (SCIRP) is one of the largest Open Access journal publishers. It is currently publishing more than 200 open access, online, peer-reviewed journals covering a wide range of academic disciplines. SCIRP serves the worldwide academic communities and contributes to the progress and application of science with its publication.

Other selected journals from SCIRP are listed as below. Submit your manuscript to us via either submit@scirp.org or Online Submission Portal.
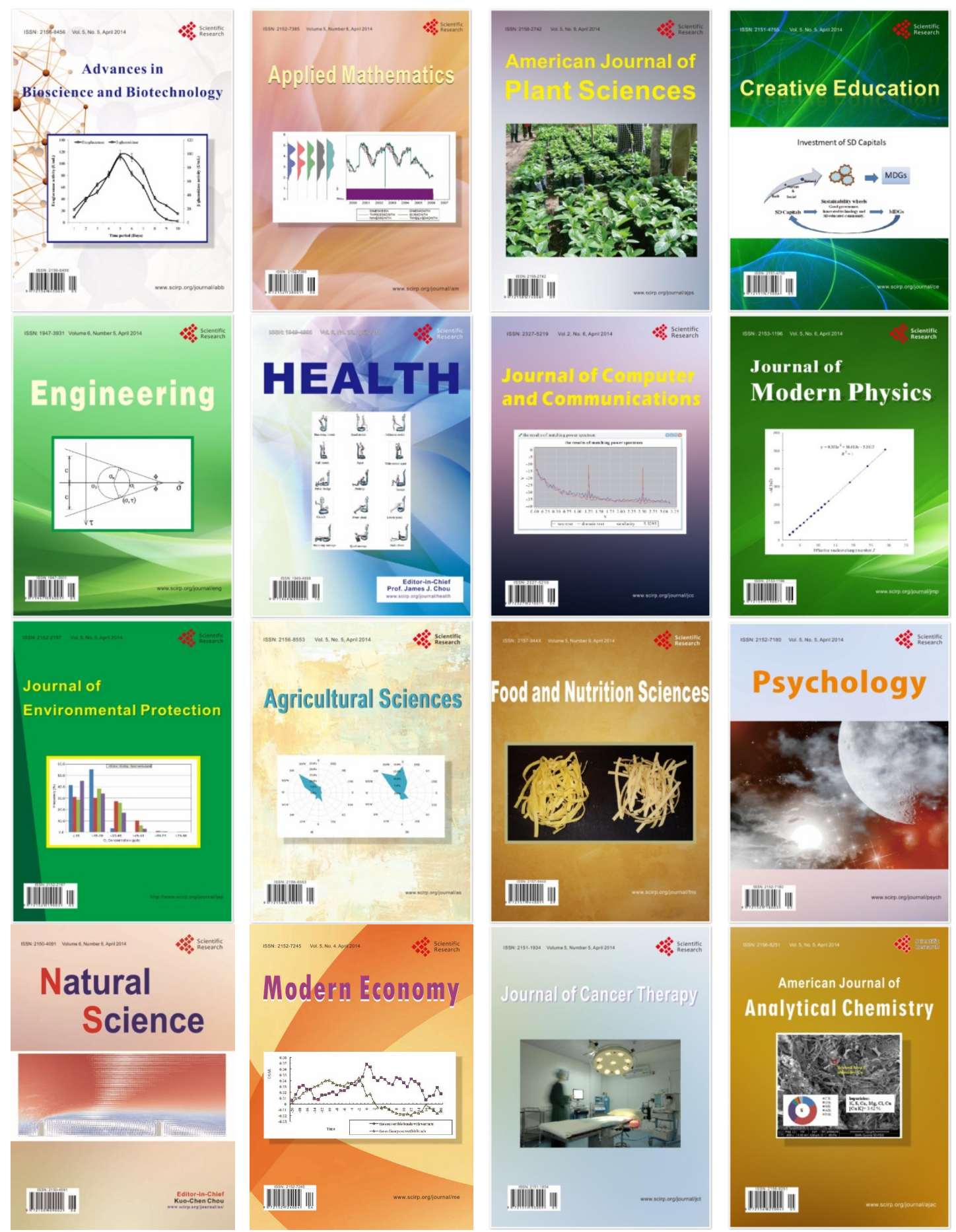